\title{
Structure and Magnetic Properties of Sm/Fe Multilayers Versus Substrate Temperature
}

\author{
X.X.Zhang, C. Ferrater, R. Zquiak and J. Tejada \\ Fac. Fisica, Universitat re Barcelona, Diagonal 647, 08028 \\ Barcelona (SPAIN)
}

\begin{abstract}
Three $\operatorname{Sm}(2 \AA) / \mathrm{Fe}(3 \AA)$ multilayers have been made using two electron beams in a high vacuum chamber onto very thin Kapton foils at different substrate temperatures, $\left(\mathrm{T}_{\mathrm{s}}=40^{\circ} \mathrm{C}, 150^{\circ} \mathrm{C}\right.$ and $\left.230^{\circ} \mathrm{C}\right)$, with the same total thickness of $3000 \AA$. We have found that the substrate temperature strongly affects structure and magnetic properties of the samples. For a substrate temperature of $150^{\circ} \mathrm{C}$ the sample behaves as a three dimensional random magnet.
\end{abstract}

\section{INTRODUCTION}

Magnetic multilayers show many interesting phenomena depending on their thickness, elements and growth conditions such as the nature of substrate, deposition rate and substrate temperature etc. Also the interfaces have an enormous influence on the magnetic properties of multilayers. When the single layers are thin enough, the multilayer can be seen as an alloy [1]. Due to the presence of the RE (rare earth) metals which have very large magnetic anisotropy, an amorphous alloy formed at interfaces between $\mathrm{RE}$ and TM (transition metal) may behave as random ferromagnets [1-3]

RE-TM random magnets are characterized by strong ferromagnetic exchange and random magnetic anisotropy. Such systems have been successfully described within the correlation spin-glass model [3]. According to this model, the magnetization is favorable to a stochastic pattern with a ferromagnetic correlation length, $R_{f}$, which depends on the exchange, $A\left(\mathrm{erg} / \mathrm{cm}^{3}\right)$, the strength of the random anisotropy, $K\left(\mathrm{erg} / \mathrm{cm}^{3}\right)$, and the length $R_{a}$ at which anisotropy axes are correlated. Therefore random ferromagnets may be pictured as having been formed by magnetic clusters.

In our experiments, the multilayers were made of very thin single layers of RE and TM metals in order to get the sample formed mainly by interfaces. We also found that the interfaces strongly depend on the substrate temperature.

\footnotetext{
Manuscript received May 31, 1993
}

\section{EXPERIMENTAL}

The films were deposited onto very thin Kapton foils with two electron beam evaporators in a vacuum chamber $\left(\approx 10^{-7}\right.$ Torr $)$ at different substrate temperatures. The samples correspond to the following composition: $[\mathrm{Fe}(3 \AA)$ $150,230^{\circ} \mathrm{C}$. The evaporation rate of the materials was $0.5 \mathrm{i} / \mathrm{s}$, measured by a quartz crystal oscillator. The structure of samples wa. examined by X-ray diffraction $(\mathrm{Cu}-$ $\mathrm{K} \alpha$ ). Magnetic properties were measured by Mössbauer spectroscopy and a SHE-SQUID in the temperature range from $5 \mathrm{~K}$ to $300 \mathrm{~K}$ at applied fields up to 5.4 Teslas.

\section{RESULTS AND DISCUSSION}

The structure of the samples was analyzed by $\mathrm{X}$-ray diffraction $(\mathrm{Cu}-\mathrm{K} \alpha)$. It was found that sample $1, \mathrm{~T}_{\mathrm{S}}=40^{\circ} \mathrm{C}$, was formed by amorphous SmFe clusters and polycrystalline $\alpha$-Fe; sample $2, \mathrm{~T}_{\mathrm{S}}=150^{\circ} \mathrm{C}$, was only formed by amorphous $\mathrm{SmFe}$ clusters, due to the temperature enhanced interdiffusion. As $\mathrm{T}_{S}$ increased to $230^{\circ} \mathrm{C}$, sample 3 , the film was mainly constituted by polycrystalline $\alpha-\mathrm{Fe}$. In this case the atoms of the film have enough kinetic energy to move or rearrange themselves to reach a low energitical state, and consequently more and larger polycrystalline $\alpha$-iron clusters were formed.

The Mössbauer spectra were recorded at room temperature for three samples with the $\gamma$-ray perpendicular to the film plane. For sample 1, the magnetization is in plane corresponding to the relative intensity ratio: $3: 4: 1: 1: 4: 3$ and hyperfine field $\mathrm{H}_{\mathrm{hf}}=329 \mathrm{kOe}$. For sample 2 , the relative intensity changed to: $3: 1.2: 1: 1: 1.2: 3$ with the hyperfine field $\mathrm{H}_{\mathrm{hf}}=325 \mathrm{kOe}$ corresponding to amorphous $\mathrm{SmFe}$ alloy clusters with a weak perpendicular anisotropy. As $T_{S}$ increased to $230^{\circ} \mathrm{C}$, the magnetization is randomly orientated with the relative intensity $3: 2: 1: 1: 2: 3$ and the 
$\mathrm{H}_{\mathrm{hf}}=330 \mathrm{kOe}$, indicating polycrystalline $\alpha-\mathrm{Fe}$.

The low and high field magnetic measurements for sample 1 and sample 3 are in full agreement with the existence of $\alpha$-iron clusters.

The zero field cooled and field cooled magnetization of sample 2 shows clearly a maximum centred at $\mathrm{T} \approx 150 \mathrm{~K}$ corresponding to the blocking of the magnetization vector of the SmFe clusters.

Fig. I shows the isothermal magnetization of sample 2 at $5 \mathrm{~K}$ with applied field in plane. Due to the amorphous characi $r$ of the RE-TM alloy, its magnetization law should be studied by random magnet theory $[1-3]$. The magnetization law at the approach of saturation is entirely determined by the random anisotropy effect, leading to certain predictions on the dimensionality of the system [3]

Three dimensional

$$
\begin{array}{ll}
\frac{\Delta \mathrm{M}}{\mathrm{M}_{0}} \propto \frac{1}{\sqrt{\mathrm{H}}} & \mathrm{H}<<\mathrm{H}_{\mathrm{ex}} \\
\frac{\Delta \mathrm{M}}{\mathrm{M}_{0}} \propto \frac{1}{\mathrm{H}^{2}} & \mathrm{H}>>\mathrm{H}_{\mathrm{ex}}
\end{array}
$$

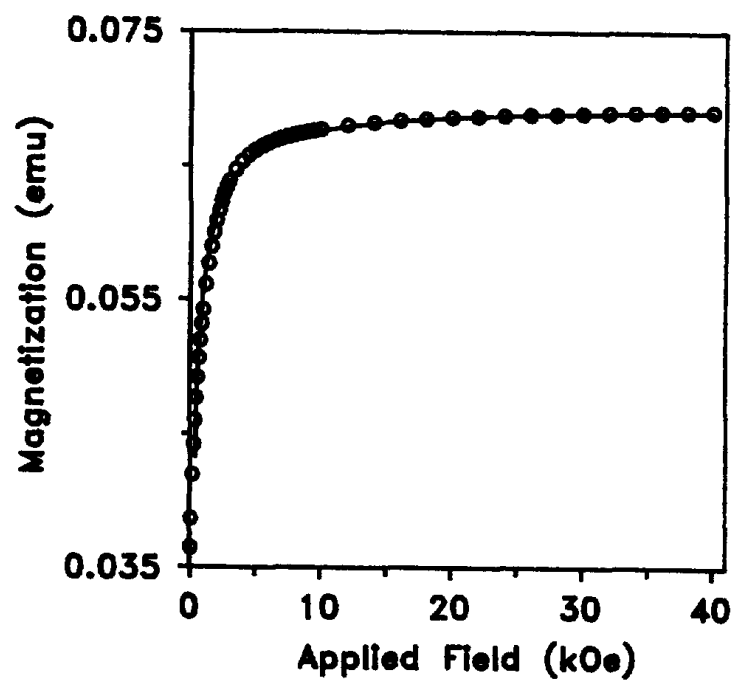

Fig.1 Magnetization vs applied field at $\mathrm{T}=5 \mathrm{~K}$ for sample 2 (points); fitting results using Eq. (3) with correlation function $C(x)=\exp \left(-x^{2}\right)($ line $)$.
Two dimensional

$$
\begin{array}{ll}
\frac{\Delta \mathrm{M}}{\mathrm{M}_{0}} \propto \frac{1}{\mathrm{H}} & \mathrm{H} \ll<\mathrm{H}_{\mathrm{ex}} \\
\frac{\Delta \mathrm{M}}{\mathrm{M}_{0}} \propto \frac{1}{\mathrm{H}^{2}} & \mathrm{H} \gg \mathrm{H}_{\mathrm{ex}}
\end{array}
$$

Where $\mathrm{H}_{\mathrm{ex}}=2 \mathrm{~A} /\left(\mathrm{M}_{0} \mathrm{R}_{\mathrm{a}}{ }^{2}\right)$. The magnetization data $\mathrm{M}(\mathrm{H})$ of sample 2 were well fitted by the $1 / \sqrt{\mathrm{H}}$ law at low magnetic field regime ( 0.5 to $3 \mathrm{kOe})$, Fig. 2 (a) and by $1 / \mathrm{H}^{2}$ law at high magnetic field regime (20kOe to $40 \mathrm{kOe}$ ) Fig.2(b).
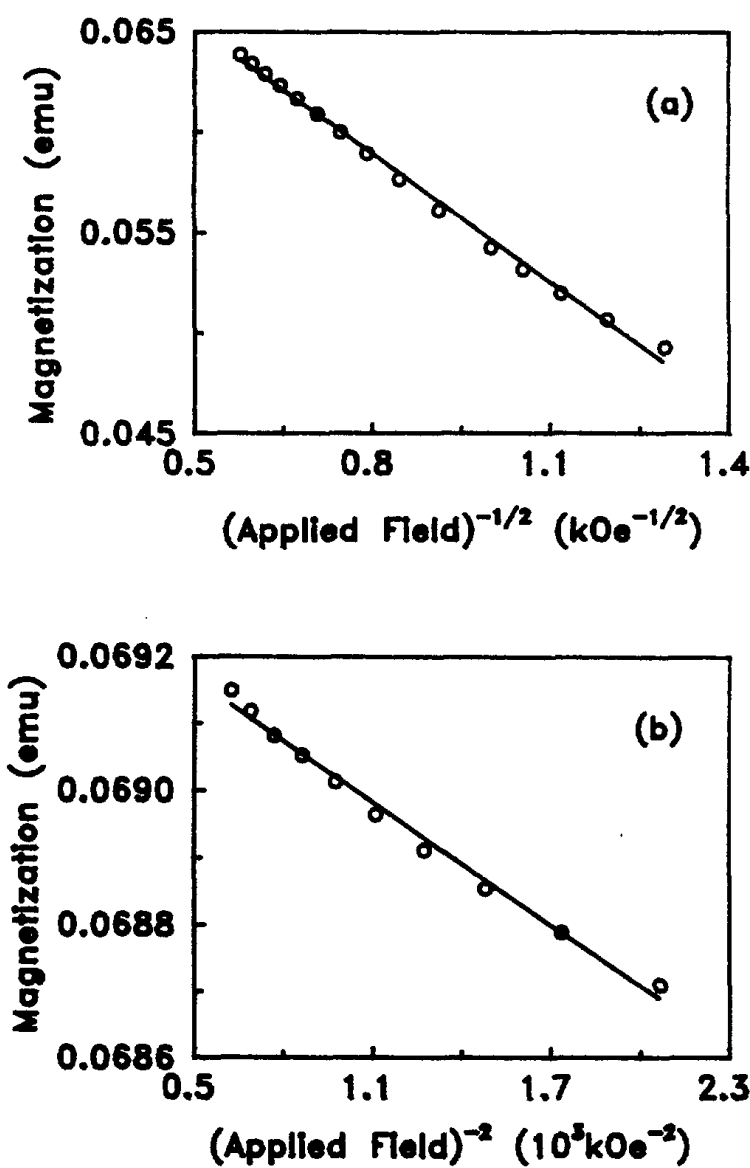

Fig. 2 (a) Low field regime in approaching to magnetization saturation for sample 2; (b) High field regime in approching to magnetization saturation for sample 2 . 
Let $S_{1 / 2}$ and $S_{2}$ be the slopes of $M$ versus $\mathrm{H}^{-1 / 2}$ and $\mathrm{H}^{-2}$ for the low field and high field regimes respectively. The magnetization law for the whole magnetic field regime can be presented as Eq. (3) [4]:

$M(H)=M_{0}-\frac{S_{1 / 2}}{\omega \sqrt{H}} \int_{0}^{\infty} d x C(x) x^{2} \exp \left[-x\left(\frac{2 S_{1 / 2}}{\omega S_{2}}\right)^{1 / 3} \sqrt{H}\right]$

where $C(x)$ is structure disorder correlation function, and $\omega \equiv \int d^{2} x C(x)[4]$. For the intermediate field regime $H \approx$ $H_{e x}$, the magnetization law is very sensible to $C(x)[2]$.

This formula has been used to extract $C(x)$ from the magnetization law in DyFeB amorphous alloy [4]. In Fig. 1, we show the fit of the magnetization curve using $\mathrm{C}(\mathrm{x})=$ $\exp \left(-\mathrm{x}^{2}\right)$ (line). From this fit, we got $\mathrm{H}_{\mathrm{ex}}=10.2 \mathrm{kOe}$.

The different properties of these samples are due to the different substrate temperature, which affects strongly the mechanism of film growth during film deposition process. Our SmFe alloy formed at interfaces behaves as a three dimensional random magnet.

\section{ACKNOWLEDGMENT}

X.X. Zhang thanks "DGICYT" for financial support.

\section{REFERENCES}

[1] X.X. Zhang, R. Zquiak, J.R. Ruiz and J. Tejada "Magnetic behavior of $\mathrm{Y} / \mathrm{Fe}$ multilayers" J. Magn. Magn. Matter Vol. 118, pp 70.76, June, 1993.

[2] J.M. Ruiz, X.X. Zhang, O. Iglesias, A. Garcias and J. Tejada, "Sturcture disorder in two-dimensional random magnet: very thin films of rare earth and transition metals" Phys. Rev. B Vol. 47, May, 1993.

[3] E.M. Chudnovsky, W.M. Saslow and R.A. Serota, "Ordering in ferromagnets with random anisotropy" Phys. Rev. B, Vol. 33, pp 251-261,

Jan. 1986; E.M. Chudnovsky," Magnetic properties of amorphous ferro- magnets (invited)" J. Appl. Phys. Vol. 64, pp 5770-5775, Nov. 1988.

[4] J. Tejada, B. Martinez, A. Labarta and E.M. Chudnovsky, "Correlated spin glass generated by structure disorder in the amorphous $\mathrm{Dy}_{6} \mathrm{Fe}_{74} \mathrm{~B}_{20}$ alloys" Phys. Rev. B, Vol 44, pp 7698-7700, Oct. 1991. 